\title{
Erratum to: Flux Tubes, Field Configuration and Non-Perturbative Dynamics of QCD
}

\author{
Deependra Singh Rawat, H. C. Chandola, H. C. Pandey and D. Yadav
}

\section{Erratum to:}

Chapter "Flux Tubes, Field Configuration and Non-Perturbative Dynamics of QCD" in:

Md. Naimuddin (ed.), XXII DAE High Energy Physics

Symposium, Springer Proceedings in Physics 203, https://doi.org/10.1007/978-3-319-73171-1_147

The original version of the book was inadvertently published with incorrect author name and affiliations in Chapter 147, which have to be corrected as follows:

In the first occurence, the author name "H. C. Pandey" has to be changed as "H.C. Chandola" and the corresponding email id "hempandey@birlainstitute.co.in" has to be replaced with "chandolaharish@gmail.com".

In the second occurence, the email id "chandolaharish@gmail.com" has to be replaced with "hempandey@ birlainstitute.co.in" for author "H. C. Pandey".

And also, a new acknowledgement text has to be included at the end of the chapter. The erratum chapter and the book have been updated with the changes.

The updated online version of this chapter can be found at https://doi.org/10.1007/978-3-319-73171-1_147 